\title{
Introducing an ambulatory PE pathway with no extra resource
}

\author{
Authors: Wendy Baird, ${ }^{\mathrm{A}^{*}}$ Emily Hannah, ${ }^{\mathrm{B}}$ Mark Feenan, ${ }^{\mathrm{B}}$ Chiara Byrne, ${ }^{\mathrm{B}}$ Mary Burke, ${ }^{\mathrm{B}}$ Joanne Dougan, ${ }^{\mathrm{B}}$ \\ David Haaijer $^{B}$ and Judith Smyth ${ }^{B}$
}

\section{Introduction}

New or suspected diagnosis of pulmonary embolism (PE) is a common cause of presentation to the acute medical take with a UK annual incidence of $60-70$ per 100,000 population. Recent studies have demonstrated that approximately $37-44 \%$ of new diagnosis of PE could be classified as low risk using the PESI scoring system and safely managed as an outpatient. This project aimed to develop an ambulatory PE service within a busy district general hospital serving a population of 360,000 with no ambulatory unit and no extra resource.

\section{Materials and methods}

A retrospective case review was carried out on all medical admissions in the 12-month period from November 2015-October 2016 who had a coded discharge diagnosis of confirmed PE. Eligibility for ambulatory care was retrospectively applied using a validated tool (PESI score) calculated based on admission observations and patient past medical history. Patients who had a high PESI score or other contraindications to ambulatory management (eg right heart strain, significant comorbidities) were excluded.

This showed that there were 93 confirmed PE cases in the 12-month period. Of these, 38 patients would have met eligibility criteria for consideration for ambulatory management. The average length of stay was 6 days for these patients, equating to a potential saving of 268 bed days per year with a cost saving of $£ 139,360$. This does not include admissions for suspected PE who subsequently had a negative computed tomography pulmonary angiography (CTPA).

Following this review, we set up a collaborative working group inclusive of representatives of emergency department, acute medical team, administration staff, radiology and pharmacy. A PE pathway was established using current British Thoracic Society guidelines using existing 'chair' space in the clinical decision unit within the emergency department for an initial 2-week pilot.

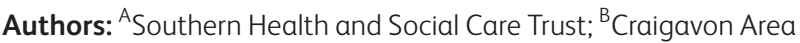
Hospital

${ }^{*} \mathrm{RCP}$ chief registrar

\section{Results and discussion}

Five patients were ambulated during the 2-week pilot phase. This resulted in a cost saving of approximately $£ 3,640$ in bed days saved for this group. This has demonstrated the cost-effectiveness of the ambulatory pathway and as a result we intend to make this a permanent service within our trust. The patient feedback for this group has been positive and we are monitoring long-term outcomes from this pathway.

\section{Conclusion}

Collaborative working across specialties has led to the development of a new ambulatory PE service with no added cost. We hope to develop our ambulatory service further with the aim of reducing medical admission rates and improve quality of care for our local population.

\section{Reference}

1 Howard LSGE, Barden S, Condliffe R et al. British Thoracic Society guideline for the initial outpatient management of pulmonary embolism (PE). Thorax 2018;73:ii1-29. 